\title{
Content Based Image Retrieval Using Discrete Curvelet Transform
}

\author{
Ms.Kamala. $\mathrm{k}^{1}$, Mr. P. S. S. Chakravarthi ${ }^{2}$, Dr. M. Venugopala Rao ${ }^{3}$ \\ ${ }^{I}$ (Student,Digital Electronics and Communcation Systems,Narasaraopeta Engineering College/JNTU \\ Kakinada,India) \\ ${ }_{2}^{2}$ (Professor,Eelectronics and Communcation Engineering,Narasaraopeta Engineering College/JNTU \\ Kakinada,India) \\ ${ }_{3}^{3}$ (Professor,Eelectronics and Communcation Engineering,KL University,India)
}

\begin{abstract}
Feature extraction is a key issue in contentbased image retrieval (CBIR). In the past, a number of texture features have been proposed in literature, including statistic methods and spectral methods. However, most of them are not able to accurately capture the edge information which is the most important texture feature in an image. Recent researches on multi-scale analysis, especially the curvelet research, provide good opportunity to extract more accurate texture feature for image retrieval. Curvelet was originally proposed for image denoising and has shown promising performance. In this paper, a new image feature based on curvelet transform has been proposed. We apply discrete curvelet transform on texture images and compute the low order statistics from the transformed images. Images are then represented using the extracted texture features. Retrieval results show, it significantly outperforms the widely used Gabor texture feature.
\end{abstract}

Keywords - texture, image retrieval, curvelet transform, CBIR, Gabor filters.

\section{Introduction:}

Nowadays, due to the availability of large storage spaces, huge number of images have been produced and stored around the world. With this huge image database, people want to search it and make use of the images in it. Here comes the challenge of image retrieval and researchers try to find out accurate ways of searching images. Basically, images can be retrieved in two ways, firstly, text based and secondly, contentbased or query by example based. Text based retrieval approach is very well known and widely used. In this process users are provided a text area to enter the key words on the basis of which image searching is done. It is widely used in Google web based image searching technique. Though the concept is very familiar to us, this approach has two notable drawbacks. (1) The images in the database are annotated manually by annotators using key words, which is a very time consuming process for a large database. (2) The retrieval solely depends on the human perception based text annotation. The consequence is that there is significant inconsistency in understanding of image content by different annotators, so the key words vary a lot and retrieval results are usually very poor. To avoid the above mentioned problem, the second approach, Content-based image retrieval has been proposed by the researchers. The term CBIR seems to have originated in the early 1990's. Since then it is an ongoing process. Different from text based image search, CBIR techniques use low-level features like texture, color, and shape to represent images and retrieves images relevant to the query image from the image database. Among those low level image features, texture feature has been shown very effective and subjective. A variety of techniques have been developed for extracting texture features. These techniques can be broadly classified into spatial methods and spectral methods. In spatial approach, most techniques rely on computing values of what are known as low order statistics from query and stored images. These methods compute texture features such as the degree of contrast, coarseness, directionality and regularity or periodicity, directionality and randomness . Alternative methods of texture analysis for image retrieval include the use of Gabor filters, wavelet and DCT . Statistic techniques suffer from insufficient number of features and sensitive to image noise. The spectral methods in literature, however, do not capture edge information accurately. In this paper, a new texture feature based on curvelet transform is proposed. The technique makes use of curvelet transform which represents the latest research result on multiresolution analysis. By combining the advantages of the two methods, image edge information is captured more accurately than conventional spectral methods such as wavelet and Gabor filters. Curvelet was originally proposed for image denoising and has shown promising performance. As it captures edge and linear information accurately, it has also shown promising results in character recognition recently. $\mathrm{Ni}$ and Leng attempted an initial application of curvelet on color image retrieval, but it was not implemented properly and no meaningful result was reported. In their work, no benchmark image database is used, no retrieval accuracy was reported and there was no comparison with other techniques .In this paper, we describe the theory and implementation of curvelet, apply curvelet transform to a standard image database, and compare its retrieval result with the best texture features in literature-Gabor filter feature and wavelet feature. 
The rest of the paper is organized as following. In section II., we describe the curvelet transform. In section III, curvelet feature extraction technique is described. Experimental and comparison results will be shown in section IV. Section V. concludes the paper.

\section{Description Of Curvelet Transform}

Basically, curvelet transform extends the ridgelet transform to multiple scale analysis. Therefore, let's start from the definition of ridgelet transform. Given an image function $f(x, y)$, the continuous ridgelet transform is given as

$$
\Re_{f}(a, b, \theta)=\iint \psi_{a, b, \theta}(x, y) f(x, y) d x d y
$$

Where $a>0$ is the scale, $b \epsilon R$ is the translation and $\theta \epsilon[0,2 \pi)$ is the orientation. The ridgelet is defined as:

$$
\psi_{a, b, \theta}(x, y)=a^{-\frac{1}{2}} \psi\left(\frac{x \cos \theta+y \sin \theta-b}{a}\right)
$$

Fig. 1 shows a typical ridgelet [13]. It is oriented at an angle $\theta$, and is constant along lines: $x \cdot \cos \theta+y \cdot \sin \theta=$ const. It can be seen that a ridgelet is linear in edge direction and is much sharper than a conventional sinusoid wavelet.For comparison, the 2-D wavelet is given as:

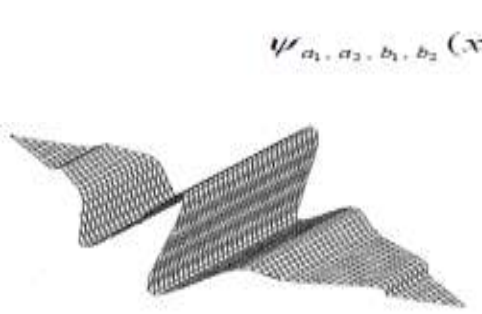

Fig. 1. A ridgelet waveform.

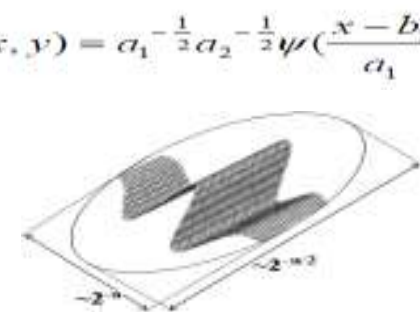

Fig. 2. Left: a single curvelet. Right: curvelets tuned to different scales and orientations

As can be seen, the ridgelet is similar to the 2-D wavelet except that the point parameters $(b 1, b 2)$ are replaced by the line parameters $(b, \theta)$. In other words, the two transforms are related by:

Wavelet: $\psi$ s cale, point-position

Ridgelet: $\psi$ scale, line-position

This means that ridgelet can be tuned to different orientations and different scales to create the curvelets, in the similar way to Gabor filters. Fig. 2 shows a single curvelet and the curvelets tuned to two scales and different number of orientations at each scale. But different from Gabor filters which only cover part of the spectrum in the frequency domain, curvelets have a complete cover of the spectrum in frequency domain. That means, there is no loss of information in curvelet transform in terms of capturing the frequency information from images. Fig. 3 (top image) shows the curvelet tiling and cover of the spectrum of a $512 \times 512$ images with 5 scales. The shaded wedge shows the frequency response of a curvelet at orientation 4 and scale 4 . It can be seen, the spectrum cover by curvelets is complete. In contrast, there are many holes in the frequency plan of Gabor filters (Fig. 3 bottom).

\section{Curvelet Feature Extraction}

The digital curvelet transform is taken on a 2-D Cartesian grid $f[m, n], 0 \leq m<M, 0 \leq n<N$,

$$
C^{D}(a, b, \theta)=\sum_{\substack{0 \leq m \leq \mathcal{M} \\ 0 \leq n=N}} f[m, n] \psi_{a, b, e}^{D}[m, n]
$$

The digital curvelet transform is implemented using the fast discrete curvelet transform. Basically, it is computed in the spectral domain to employ the advantage of FFT. Given an image, both the image and the curvelet are transformed into Fourier domain, then the convolution of the curvelet with the image in spatial domain becomes the product in Fourier domain.
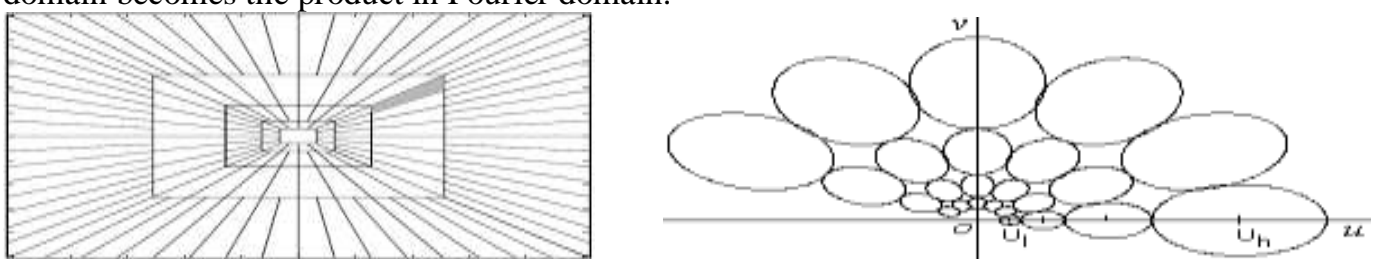

Fig. 3. left: the tiling of frequency plan by curvelets. Right: the tiling of half frequency plan by Gabor filters, the ovals are the covered spectrum. 
Finally the curvelet coefficients are obtained by applying inverse Fourier transform on the spectral product. But due to the frequency response of a curvelet is a nonrectangular wedge, the wedge needs to be wrapped into a rectangle to perform the inverse Fourier transform. The wrapping is done by periodic tiling of the spectrum using the wedge, and then collecting the rectangular coefficient area in the centre. Through this periodic tiling, the rectangular region collects the wedge's corresponding portions from the surrounding periodic wedges. The complete feature extraction process using a single curvelet is illustrated in Fig. 4.

Once the curvelet coefficients have been obtained, the mean and standard deviation are computed as the texture features for the curvelet. That is, for each curvelet, two texture features are obtained. If $n$ curvelets are used for the transform, $2 n$ texture features are obtained. A $2 n$ dimension texture feature vector is used to represent each image in the database for image retrieval. This feature extraction is applied to each of the images in the database. At the end, each image in the database is represented and indexed using its curvelet feature vector. Given a query, its curvelet features are computed using the process shown in Figure 4. The system then compares the query feature vector with all the feature vectors in the database using L2 distance:

$$
d(\mathbf{Q}, \mathbf{T})=\left(\sum_{i=0}^{2 n-1}\left(Q_{i}-T_{i}\right)^{2}\right)^{\frac{1}{2}}
$$

Where $\mathbf{Q}=\{Q 0, Q 2, \ldots, Q 2 n-1\}$ is the feature vector of the query image, and $\mathbf{T}=\{T 0, T 2, \ldots, T 2 n-1\}$ is the feature vector of target image in the database. Finally, the images in the database are ranked according to their distance $d$ to the query image, and the ranked list of images is returned to the user. This retrieval process is shown in Fig. 5.
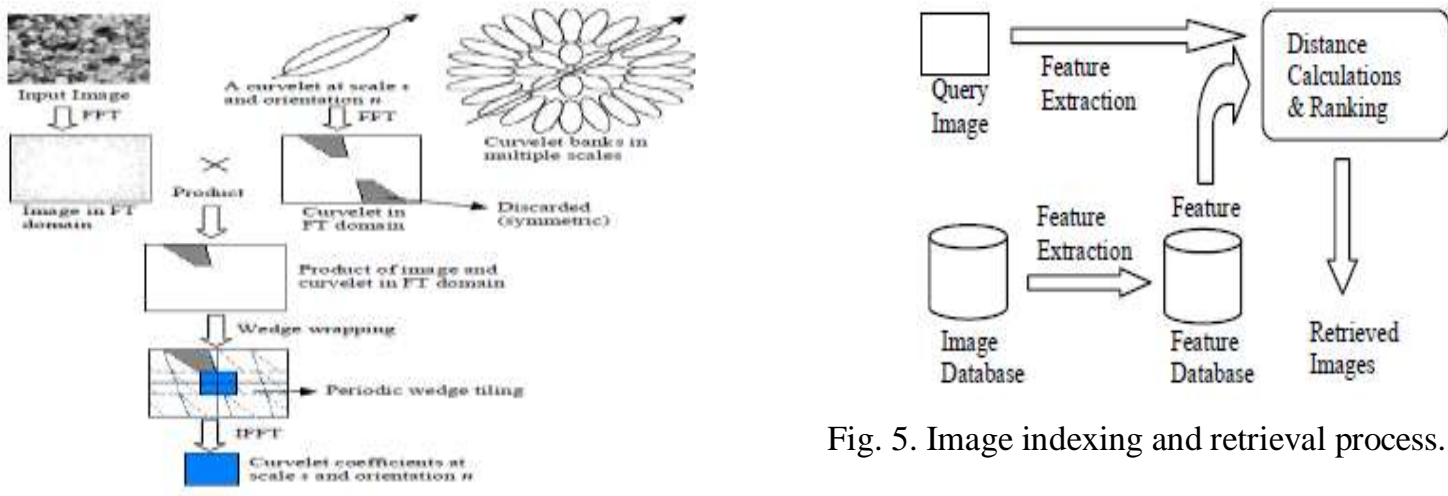

Fig. 5. Image indexing and retrieval process.

Fig. 4. Fast discrete curvelet transform.

\section{Retrieval Performance Test}

In this section, we test the performance of the curvelet feature in terms of retrieval accuracy. The widely used standard Brodatz texture database is used in the test. The database consists of 112 different categories of natural and man made texture images. Each category includes 16 similar images, each image has the same size of $128 \times 128$ pixels. In total, the image database consists of 1,792 images from 112 categories. We apply the curvelet feature extraction process described in Section III to each of the 1,792 images in the database and index each of the images using the curvelet feature vector. For the discrete curvelet transform, two tests are experimented. In the first test, images are decomposed using 4 levels curvelet transform. Based on the subband division in Figure 3, with 4 levels analysis, $50(=1+16+32+1)$ subbands of curvelet coefficients are computed. However, curvelet at angle $\theta$ produces the same coefficients as curvelet at angle $\theta+\pi$. Therefore, half of the subbands at scale 2 and 3 are discarded due to this symmetry. As the result, $26(=1+8+16+1)$ subbands are preserved, and a 52 dimension feature vector is generated for each image in the database. In the second test, images are decomposed using 5 levels curvelet transforms. Based on the subband division in Figure 3, with 5 levels analysis, $42(=1+8+16+16+1)$ subbands of curvelet coefficients are computed. Therefore, a 84 dimensionfeature vector is generated for each image in the database. The subbands distribution at each level of transform are shown in Table 1. The commonly used performance measurement, precisionrecall pair, is used for the evaluation of retrieval performance. Precision $P$ is defined as the ratio of the number of retrieved relevant images $r$ to the total number of retrieved images $n$, i.e. $P=r / n$. Precision $P$ measures the accuracy of the retrieval. Recall $R$ is defined as the ratio of the number of retrieved relevant images $r$ to the total number $m$ of relevant images in the whole database, i.e. $R=r / m$. Recall $R$ measures the robustness of the retrieval. 


$$
\begin{aligned}
& P=\frac{\text { No. of relevant images retrieved }}{\text { Total no. of tmages retrieved }} \\
& R=\frac{\text { No. of relevant images retrieved }}{\text { Total no. of relevant images in } D B}
\end{aligned}
$$

Because the ground truth is known for the whole database, every image in the database is used as the query. For each query, the precision of the retrieval at each level of the recall is obtained. These precision values are then averaged to produce the average precision-recall curve for the 1,792 database images. To benchmark the retrieval performance, we compare the curvelet retrieval result with the two best spectral texture features in literature, i.e., Gabor filter feature and wavelet feature. For Gabor filter, we use the best combinations of parameters found by Chen et al in , that is, 6 scales and 4 orientations with filter size of $13 \times 13$ pixels. The Gabor filter with this configuration generates 48 texture features for each input image, which is comparable with the 52 texture features of the 4 levels curvelet transform. The retrieval performance of curvelet feature and Gabor filter feature is shown in Fig. 6. As can be seen from the graph, the retrieval performance of curvelet feature is significantly higher than that of Gabor filter feature. The curvelet transform with 5 levels decomposition has even better retrieval performance than at 4 levels decomposition, however, at the cost of higher feature dimension which is 84 . The reasons why cuvelet feature has significantly higher retrieval performance than Gabor filter feature are due to curvelet has several advantages over Gabor filter. First, due to the half-height truncation of the filters to remove the overlap in spectral domain, many holes are created in the spectrum, that is, the spectrum is not completely covered by Gabor filters. As the result, much of the frequency information in an image is lost due to the incomplete spectrum cover. Secondly, curvelet transform uses ridgelets as the filter bank, while Gabor uses wavelet as the filter bank. Ridglets capture edge information or texture information more accurately than wavelets. Thirdly, curvelet transform is a true wavelet transform, while Gabor filter is not because it does not scale image during the filtering. Due to the scaling of image size at different levels of transform to adapt the image to the curvelets, curvelet can tolerate more scale distortions in images. This can be seen in all the examples in Fig. 7. The curvelet retrieval performance is also compared with wavelet retrieval performance on the same image database. Based on the result reported in, wavelet produces an average retrieval precision of $70.30 \%$ using the same Brodatz image database used by this paper. Curvelet, however, gives an average retrieval precision of $77.65 \%$ with 4 levels transform, and an average retrieval precision of $79.54 \%$ with 5 levels transform (see Table 2). The reasons why curvelet has better retrieval performance than wavelet are in two aspects. First, as mentioned earlier,curvelets capture more accurate edge information or texture information than wavelets. Second, as curvelets are tuned to different orientations, curvelets capture more directional features and more accurate directional features than wavelets. Fig. 7 shows a few retrieval results using both curvelet feature and Gabor filter feature. All the query images are natural textures which are difficult to retrieve. However, in all the three situations, curvelet feature gives good results and outperforms Gabor filter feature significantly.

\begin{tabular}{|l|l|l|l|l|l|}
\hline \multicolumn{7}{|c|}{ 4 level curvelet transform } \\
\hline Scale & 1 & 2 & 3 & 4 \\
\hline No. subbands & 1 & 8 & 16 & 1 \\
\hline \multicolumn{7}{|c|}{ 5level curvelet transform } \\
\hline Scale & 1 & 2 & 3 & 4 & 5 \\
\hline No. subbands & 1 & 8 & 16 & 16 & 1 \\
\hline
\end{tabular}

Table 1:Subbands Distribution At

\begin{tabular}{|l|l|l|}
\hline \multirow{2}{*}{ Recall (\%) } & \multicolumn{2}{|c|}{ Predision (\%) } \\
\cline { 2 - 3 } & $\begin{array}{l}\text { Curvelet with 4 } \\
\text { levels analysis }\end{array}$ & $\begin{array}{l}\text { Curvelet with 5 } \\
\text { Ieveb analysis }\end{array}$ \\
\hline 10 & 100 & 100 \\
\hline 20 & 93.34 & 93.95 \\
\hline 30 & 90.67 & 91.76 \\
\hline 40 & 85.38 & 86.15 \\
\hline 50 & 80.27 & 81.96 \\
\hline 60 & 72.46 & 79.01 \\
\hline 70 & 72.00 & 74.16 \\
\hline 80 & 68.72 & 71.50 \\
\hline 90 & 59.86 & 63.32 \\
\hline 100 & 48.83 & 53.57 \\
\hline Average & 77.65 & 79.54 \\
\hline
\end{tabular}
Each scale

Table 2: Retreival Accuracy Of Curvelet Feature

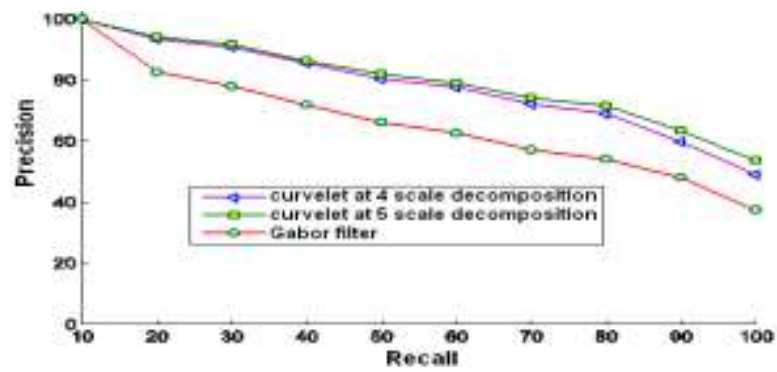

Fig. 6. Average retrieval result of 1,792 queries using both curvelet and Gabor filter. 

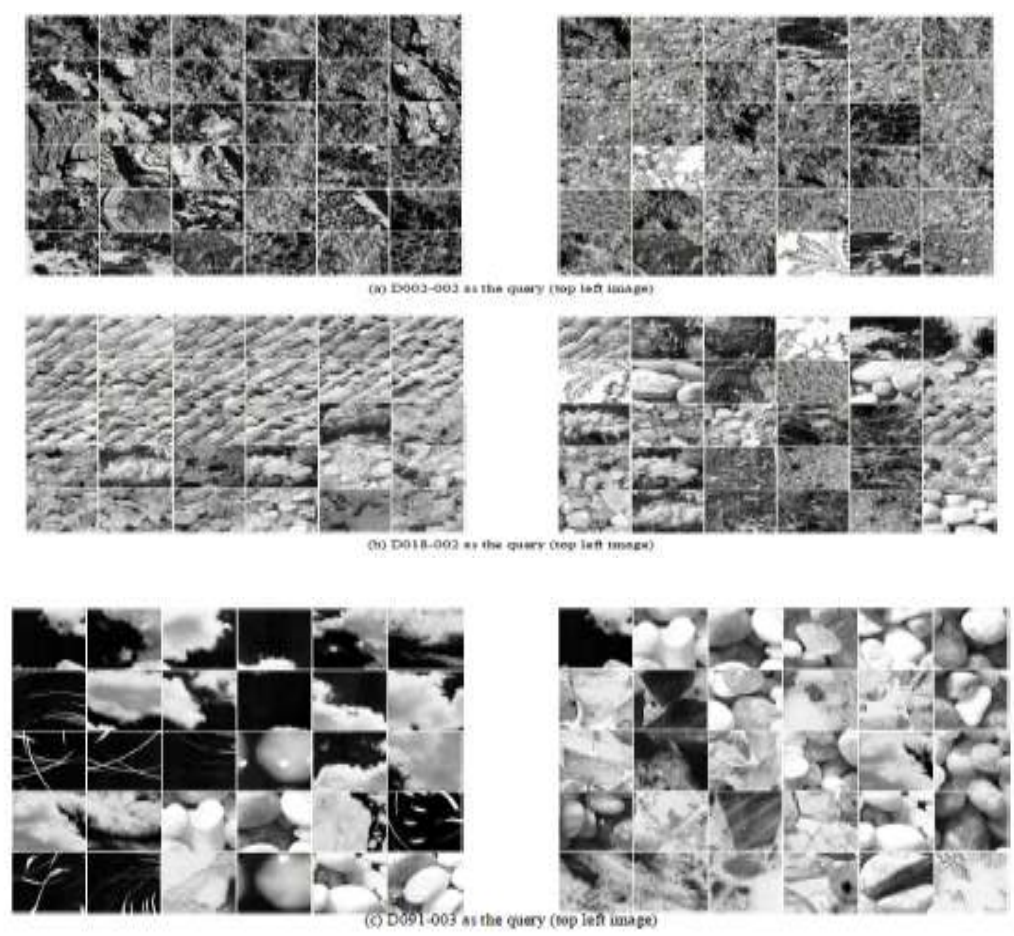

Fig. 7. First page of retrieved images by using different queries in Brodatz texture database. In all the 3 rows, the left page is the result from curvelet transform, vand the right page is the result from Gabor filters. Images in all the retrieved pages are organized from left to right and top to bottom in increased feature distance (or decreased similarity) to the query image.

\section{Conclusion}

In this paper, a new texture feature based on curvelet transform is presented. The first contribution of the paper is the systematic description, implementation, analysis and evaluation of the curvelet transform which represents the latest research progress on multi-resolution image analysis. The second contribution is the application of curvelet transform on content based image retrieval and has produced a new texture feature. Results show the curvelet texture feature is very promising for image retrieval, and significantly outperforms the best texture features in literature such as the wavelet feature and the Gabor filter feature. In future, rotation and scale invariance will be investigated to further improve curvelet retrieval performance. Application of curvelet feature in color image retrieval and semantic learning will also be investigated.

\section{References}

[1] F. Long, H. J. Zhang and D. D. Feng, Fundamentals of Content-based Image Retrieval, In Multimedia Information Retrieval and Management, D. Feng Eds, Springer, 2003.

[2] H. Tamura, S. Mori and T.Yamawaki, Texture Features Corresponding to Visual Perception, IEEE Trans. on Systems, Man and Cybernetics, 8(6): 460-473, 1978.

[3] B. S. Manjunath et al, Color and Texture Descriptors, IEEE Transactions CSVT, 11(6):703-715, 2001.

[4] F. Liu and R.W.Picard, Periodicity, Directionality, and Randomness: Wold Features for Image Modeling and Retrieval, IEEE Trans. On Pattern Analysis and Machine Intelligence,18(7):722-733, 1996.

[5] L. Chen, G. Lu, and D. S. Zhang, Effects of Different Gabor Filter Parameters on Image Retrieval by Texture, In Proc. of IEEE $10^{\text {th }}$ International Conference on Multi-Media Modelling, pp.273-278, Australia, 2004.

[6] B. S. Manjunath and W. Y. Ma, Texture Features for Browsing and Retrieval of Large Image Data, IEEE Transactions on Pattern Analysis and Machine Intelligence, 18(8):837-842, 1996.

[7] B. S. Manjunath et al, Introduction to MPEG-7, John Wiley \& Son Ltd., 2002.

[8] J. Z. Wang, J. Li, and G. Wiederhold, SIMPLIcity: Semantics-sensitive Integrated Matching for Picture Libraries, IEEE Trans. Pattern and Machine Intelligence, 23(9):947-963, 2001.

[9] Minh N. Do, Directional Multiresolution Image Representations, PhD Thesis, EPFL, 2001. [10] N. Suematsu et. al, Region-Based Image Retrieval using Wavelet Transform, Proc. $15^{\text {th }}$ International Conf. on Vision Interface, May, 2002

[10] N. Suematsu et al, Region-Based Image Retrieval using Wavelet Transform, Proc. 15th International Conf. on Vision Interface, May, 2002. 